\title{
Battery Health Determination by Subspace Parameter Estimation and Sliding Mode Control for an All-Electric Personal Rapid Transit Vehicle - The ULTra
}

\author{
C. R. Gould†, C. M. Bingham, D. A. Stone, P. Bentley \\ UNIVERSITY OF SHEFFIELD \\ Department of Electronic and Electrical Engineering \\ Mappin Street, Sheffield, S1 3JD, UK \\ Tel. +44 (0) 114 2225847, Fax. +44 (0) 1142225196 , \\ 'E-mail: c.gould@sheffield.ac.uk
}

\begin{abstract}
The paper describes a real-time adaptive battery modelling methodology for use in an all electric Personal Rapid Transit (PRT) vehicle. Through use of a sliding-mode observer and online Subspace parameter estimation, the voltages associated with monitoring the State of Charge (SoC) of the battery system are shown to be accurately estimated, even with erroneous initial conditions in both the model and parameters. In this way, problems such as selfdischarge during storage of the cells and SoC drift (as usually incurred by coulomb-counting methods due to overcharging or ambient temperature fluctuations) are overcome. Moreover, through online monitoring of the degradation of the estimated parameters, battery ageing (State of Health) can be monitored and, in the case of safetycritical systems, cell failure may be predicted in time to avoid inconvenience to passenger networks.
\end{abstract}

Due to the adaptive nature of the proposed methodology, this system can be implemented over a wide range of operating environments, applications and battery topologies, by adjustment of the underlying state-space model.

\section{INTRODUCTION}

With the development of environmentally-friendly, battery-operated vehicles and the increased use of X-bywire systems to control their operation, the demands on battery systems become of utmost importance to the operational safety and reliability of the vehicles. Large transient power demands coupled with the possibility of the battery cells being in a state of partial discharge, require that the battery management system has knowledge of the exact capability of the cells so that safe and efficient peak performance of the vehicle is achievable at all times. As an example, Figure 1 depicts example test-track transient demands on a Personal Rapid Transit (PRT) vehicle known as the ULTra (Urban Light Transport - Figure 2) developed by Advanced Transport Systems Ltd [1], which is an unmanned, steer-by-wire electric vehicle capable of carrying a $500 \mathrm{~kg}$ payload at speeds up to $40 \mathrm{kmph}$, for the purpose of improving the transportation needs of a busy city or other public space (e.g. Heathrow airport in this case) with minimal environmental impact.

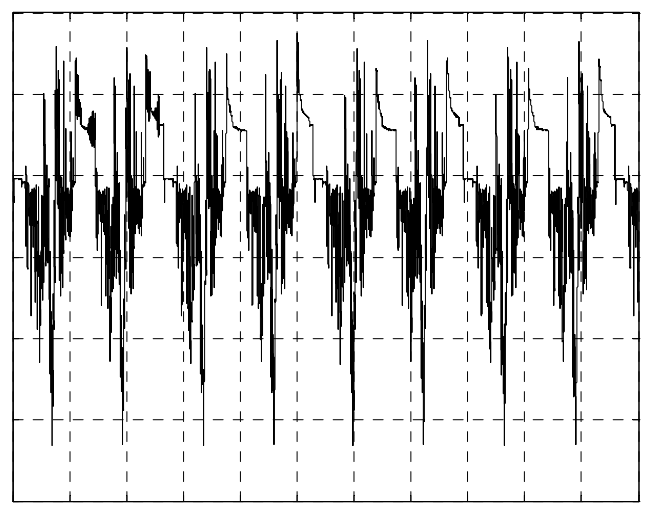

Figure 1. Example of ULTra driving cycle current-profile

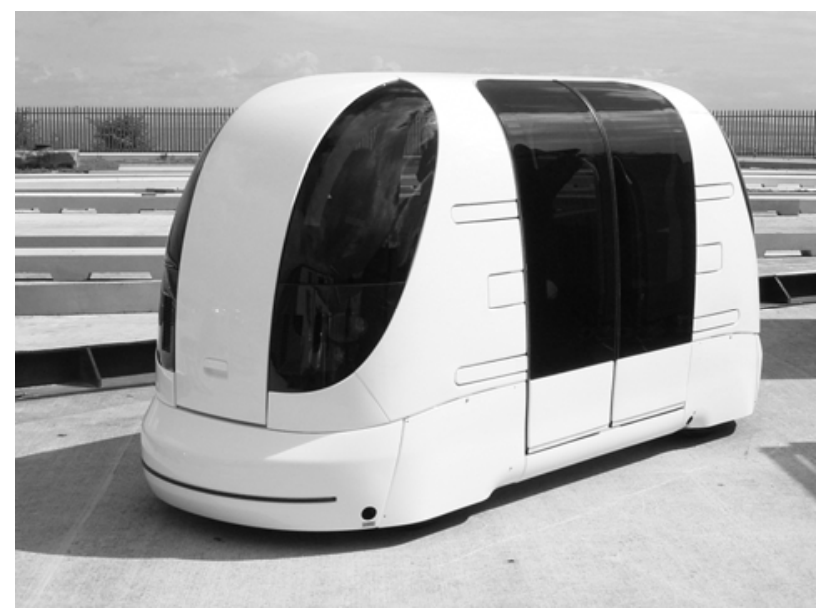

Figure 2. ULTra Personal Rapid Transit vehicle

Considering the many dynamic mechanisms occurring inside a battery to enable charge to be accepted and 
supplied [2], it is generally accepted that monitoring of the cell's State-of-Charge (SoC) to prevent dangerous overcharging, and the ability to estimate the State-of-Health (SoH) so that excessive transients may be confidently sourced/sinked without catastrophic failure of the safetycritical power-pack, is of primary importance in battery management strategies.

Whilst SoC characterisation techniques have been published for lead-acid battery technologies [5-9], $\mathrm{SoH}$ determination is less well-reported, primarily because the $\mathrm{SoH}$ is a qualitative rather than quantitative measure of the ageing of a battery $[3,4]$ and its ability to provide its nominal capacity over extended lengths of time. In most cases, the $\mathrm{SoH}$ is estimated by monitoring the degradation of specific battery model parameters, as suggested in [5], or close inspection of operational phenomena such as the Coup de Fouet technique used in [6].

The SoC of a battery is also often inferred, usually from external measurements taken during either excitation or dormancy of the cell. Many authors have investigated modelling strategies for accurate SoC estimation, such as coulomb-counting methods [7], electrochemical battery models [6], or equivalent circuit battery models $[5,8,9,10]$.

Of these, the simplest is coulomb-counting, whereby the battery is treated as a simple capacitor charge store (where the voltage across its output terminals is a function of the total integrated current demand). The simplicity of this method is, however, undermined by the need for accurate a priori knowledge of the initial capacity, and that gassing reactions caused by overcharging are not accommodated, leading to SoC 'drift'. Electrochemical models are used to predict energy storage occurring in the cells, similar to the coulomb-counting method, and often require complex models to accommodate gassing, aging or temperature effects.

More popular is the equivalent circuit model methodology, where the mechanics of charge transfer and chemical effects are modelled as circuit components, traditionally resistor-capacitor networks. The authors of $[5,8,9]$ demonstrate the estimation of static battery parameters through Kalman filter observer theory, circuit theory, and estimation filtering theory according to models based on the well-known Randles' model [10]. These result in good SoC estimation, and in the case of [5], a demonstration of SoH deterioration due to the use of closed-loop observer theory. For dynamic drive cycles described by Figure 1, however, static parameter values will not suffice to produce an accurate model in either open- or closed-loop.

This paper focuses on providing an accurate dynamic model of systems under the stresses such as the ULTra drive-cycle presents, with its large bipolar transients and requirements for occasional charge scenarios. Here it will be shown that a Subspace parameter estimation algorithm can be performed online in combination with a simple sliding-mode observer to allow adaptive identification and correction of the model voltages associated with $\mathrm{SoC}$ and $\mathrm{SoH}$ monitoring. The resulting dynamic parameter estimates will also be shown to be useful for faultprediction and battery end-of-life determination.

\section{BATTERY MODEL}

The ULTra relies on a system of four $12 \mathrm{~V}$ ValveRegulated Lead-Acid (VRLA) batteries, nominally rated between 45Ahrs (at the C5 rate) and 50Ahrs (at the C20 rate), which will be modelled individually using the Randles' model shown in Figure 3. In this model, $R_{d}$ represents the self-discharge resistance, and is usually so large that minimal current is drawn and is therefore neglected in this dynamic model, resulting in the statespace description given in (1). Classically, $C_{b}$ is considered the main charge store (also known as the 'bulk capacitance'), and the voltage across it considered a suitable indicator of SoC, whilst $\mathrm{SoH}$ deterioration can be inferred by any decrease in the value of $C_{b} . R_{i}$ models the resistance of the battery's terminals and inter-cell connections, whilst $R_{t}$ and $C_{s}$ (also known as transfer resistance and surface capacitance) describe transient effects resulting from shifting ion concentrations and plate current densities.

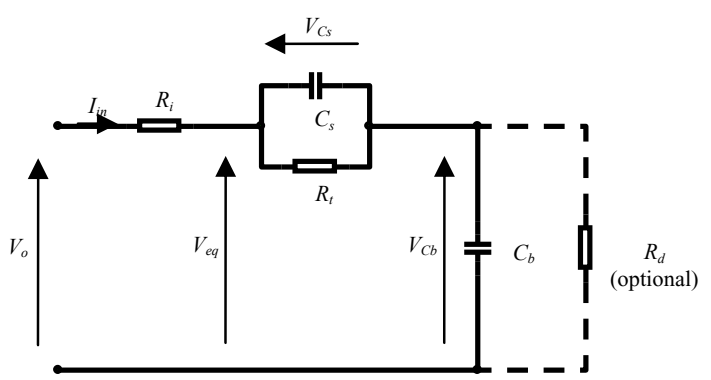

Figure 3. Randles' equivalent circuit model

$$
\begin{aligned}
& \dot{V}_{C_{b}}=I_{i n} / C_{b} \\
& \dot{V}_{C_{s}}=\left(I_{i n} R_{t}-V_{C_{s}}\right) / C_{s} R_{t} \\
& V_{o}=V_{C_{s}}+V_{C_{b}}+I_{i n} R_{i}
\end{aligned}
$$

\section{SLIDING MODE OBSERVER THEORY}

An uncertain dynamical system description (2) may be 'observed' using the discontinuous observer form (3) proposed by Utkin (1981), where $\hat{x}_{1}$ and $\hat{y}$ are the state estimates for $x_{1}$ and $y$, whilst $L \in \mathfrak{R}$ and $M \in+\Re$ are feedback gains. This results in the discontinuous observer form given in (4), where $V_{o}=V_{e q}+I_{i n} R_{i}$ :

$$
\begin{aligned}
\dot{x}(t) & =A x(t)+B u(t)+f(x, u, t) \\
y(t) & =C x(t) \\
\dot{\hat{x}}_{1} & =A_{11} \hat{x}_{1}+A_{12} \hat{y}+B_{1} u+L v_{1} \\
\dot{\hat{y}} & =A_{21} \hat{x}_{1}+A_{22} \hat{y}+B_{2} u-v_{2} \\
v_{i} & =M \operatorname{sgn}(\hat{y}-y)
\end{aligned}
$$




$$
\left[\begin{array}{c}
\dot{V}_{C_{b}} \\
\dot{V}_{C_{s}} \\
\dot{V}_{e q}
\end{array}\right]=\left[\begin{array}{ccc}
0 & 0 & 0 \\
0 & -1 / C_{s} / R_{t} & 0 \\
0 & -1 / C_{s} / R_{t} & 0
\end{array}\right]\left[\begin{array}{c}
V_{C_{b}} \\
V_{C_{s}} \\
V_{e q}
\end{array}\right]+\left[\begin{array}{c}
1 / C_{b} \\
1 / C_{s} \\
\left(C_{b}+C_{s}\right) / C_{b} / C_{s}
\end{array}\right] I_{\text {in }}+\left[\begin{array}{c}
L_{1} M \operatorname{sgn}\left(\hat{y}-V_{e q}-I_{i n} R_{i}\right) \\
L_{2} M \operatorname{sgn}\left(\hat{y}-V_{e q}-I_{i n} R_{i}\right) \\
-M \operatorname{sgn}\left(\hat{y}-V_{e q}-I_{i n} R_{i}\right)
\end{array}\right]
$$

\section{SUBSPACE PARAMETER ESTIMATION}

The input-output relationship of a discrete-time state space model with system matrices $A_{d}, B_{d}, C_{d}$ and $D_{d}$ and white noises $w(t), v(t)(5)$, can also be represented by the relationship shown in (6), for any invertible matrix ' $T$ '. It can be shown (Ljung 10.80-10.129 [11]), that the system of matrices $A_{d}, B_{d}, C_{d}, D_{d}$, and initial conditions $\tilde{x}(0)$ given sufficient input-output data, may then be estimated by solution of the resulting Subspace algorithms by a least squares method.

$$
\begin{gathered}
x(t+1)=A_{d} x(t)+B_{d} u(t)+w(t) \\
y(t)=C_{d} x(t)+D_{d} u(t)+v(t) \\
\tilde{x}(t+1)=T^{-1} A_{d} T \tilde{x}(t)+T^{-1} B_{d} u(t)+\tilde{w}(t) \\
y(t)=C_{d} T \tilde{x}(t)+D_{d} u(t)+v(t)
\end{gathered}
$$

This allows static battery model parameters to be estimated for a 'window' of input-output data $\left(V_{o}\right.$ and $\left.I_{i n}\right)$. To capture the dynamic variation of the battery parameters, a FIFO buffer is used to store the accumulation of input-output data. Due to the large timeconstants involved with $C_{b}$, it is impossible to acquire sufficient buffered data for accurate estimation of the exact value of $C_{b}$, without neglecting other, more transient parameter variations (i.e. occasional charging periods), and so a structured Subspace estimation model is used in which $C_{b}$ is assumed to be large $(100000 \mathrm{~F})$ and constant, over a small buffer of data (90 seconds). Inaccuracies in $C_{b}$ will be corrected for by the inherent predictor-corrector action of the sliding mode observer, allowing determination of $C_{b}$, and hence $\mathrm{SoH}$.

\section{RESUlts}

The observer is initialised with $L_{1}=-1, L_{2}=-0.002$, $M=0.02, \quad R_{i}=6 \mathrm{~m} \Omega, \quad R_{t}=10 \mathrm{~m} \Omega, \quad C_{s}=2000 \mathrm{~F}$, $C_{b}=100000 \mathrm{~F}, V_{C_{s}}(0)=0 \mathrm{~V}$ and an erroneous initial voltage, $V_{C_{b}}(0)=11 \mathrm{~V}$. The algorithms detailed in (1)-(6) are then used to monitor the effect that a continuous series of cycles constructed from Figure 1 has on a fully charged, well-conditioned battery. After 90s, and at regular subsequent intervals (10s in this case) the Subspace algorithms of [11] are used to adapt the battery model parameters online, resulting in the time-varying parameters shown in Figures 4, 5 and 6, demonstrating that careful monitoring of these parameters can indicate dangerous areas of operation such as overcharge/discharge situations as well as possible fault conditions such as cell short-circuits.

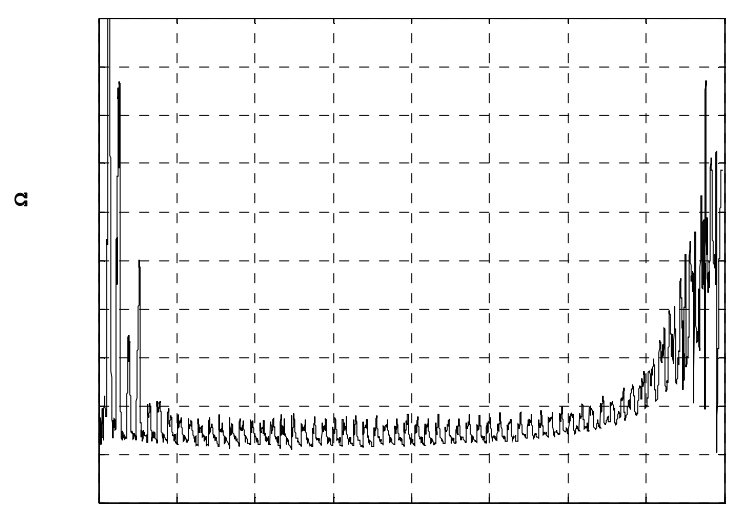

Figure 4. Online estimated battery parameters: $R_{i}$

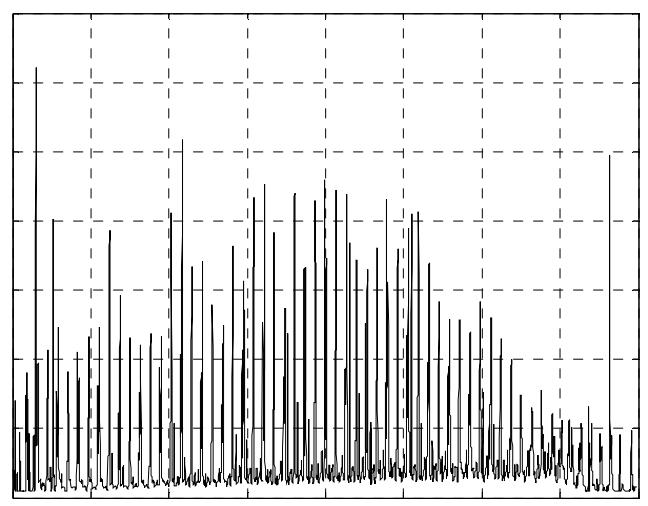

Figure 5. Online estimated battery parameters: $C_{s}$

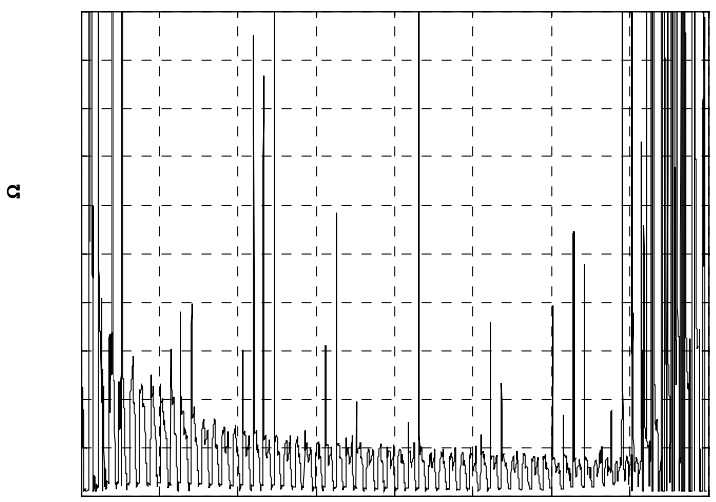

Figure 6. Online estimated battery parameters: $R_{t}$ 
The resulting observer estimate of $V_{C_{b}}$ indicating the variation of SoC, is filtered with a low-pass filter $\left(\omega_{c}=\right.$ $0.057 \mathrm{rads}^{-1}$ ), and shown in Figure 7 , demonstrating the ability of the observer to correct for erroneous initial conditions compared to an initial open-circuit voltage $\left(V_{C_{b}}\right)$ reading. Moreover, the variation in $C_{b} / \mathrm{SoH}$ (Figure 8) may be estimated by applying a linear curve-fit to buffered estimations of $V_{C_{b}}$, and by considering the integrated current through this capacitor over these sample times (typically 1800 s to provide stable results).

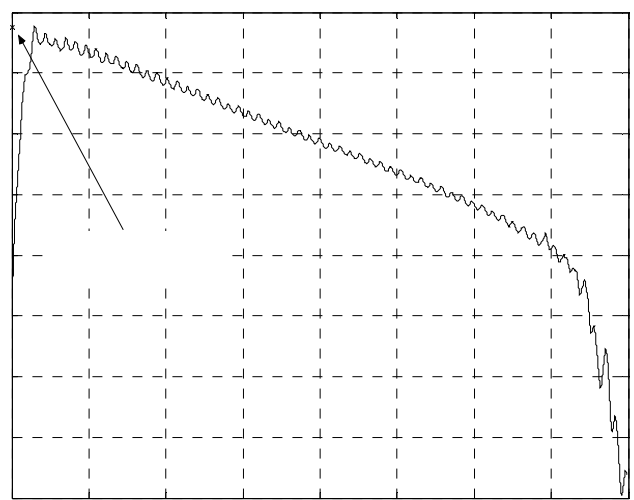

Figure 7. Estimated $V_{C b}$ showing convergence of observer to correct initial condition

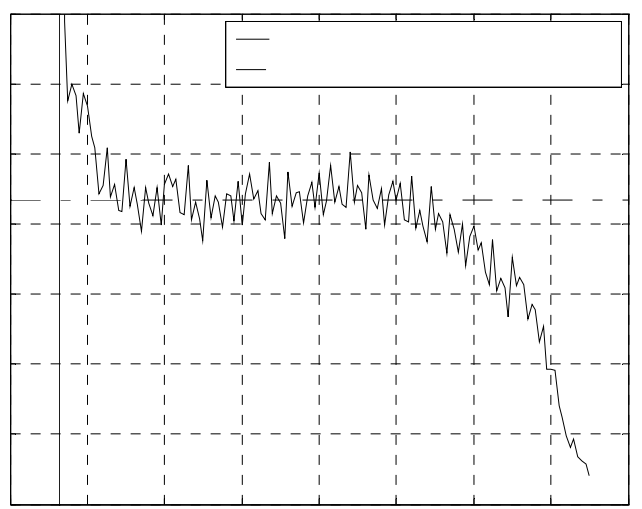

Figure 8. Resulting variation of $C_{b}$ from gradient of Figure 7 and current integration

After the initial convergence of the Subspace parameter and observer algorithms, It can be seen that the value of $C_{b}$ settles to an almost constant value (especially in the region of preferred operation occupied between $\sim 80 \%$ SoC $($ Time $=3000 \mathrm{~s})$ and $\sim 30 \%$ SoC $($ Time $=10500 \mathrm{~s})$.

Through accelerated ageing tests, whereby the battery under test is repeatedly charged (at the $\mathrm{C} 10$ rate) and then discharged with a repetition of the cycles demonstrated in Figure (1), the methodology above can be used to demonstrate the gradual degradation of SoH of the battery over time (Figure (9). This degradation can be approximated with the linear relationship described in (7)

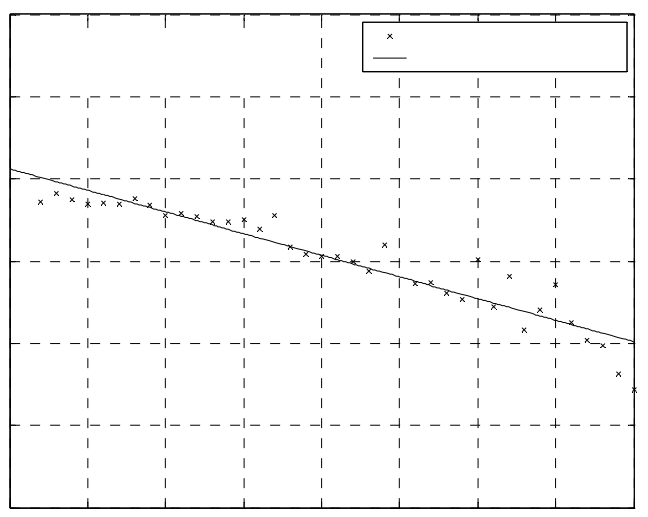

Figure 9. Decline in estimated $C_{b}$ with 'age' of battery

$$
C_{b}=-264 N+90600
$$

where $N$ is the test number.

Equivalently, as each test is implemented, the total current demand may be integrated over the test duration to estimate the total capacity of the battery under test at that time (Figure (10)). Similarly, a linear relationship (8) can also be applied.

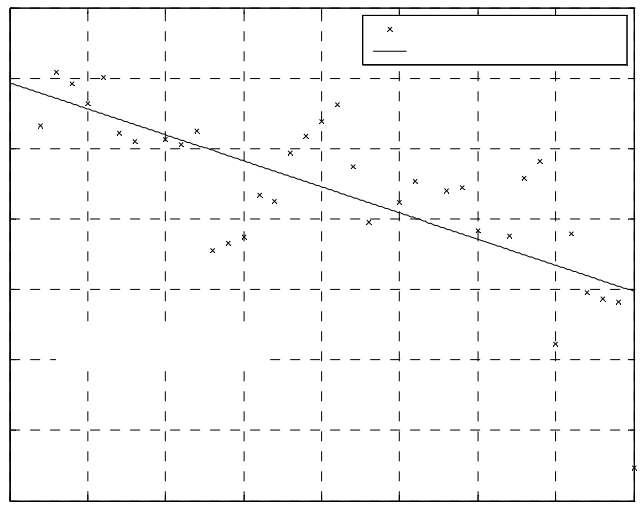

Figure 10. Reduction of available capacity with 'age' of battery

$$
\text { Ahrs }=-0.074 N+48.9
$$

The linear approximations of (7) and (8) may be manipulated to provide a relationship (9) between the total available capacity of a battery and the estimated value of $\mathrm{Cb}$ at any time during its life (Figure 11).

$$
\text { Ahrs }=0.00028 C_{b}+23.5
$$

Taking the example demonstrated in Figures (7) and (8), the estimated $C_{b}$ of $87000 \mathrm{~F}$ maps to an available capacity of 47.86Ahrs, compared to that of 48.1Ahrs of a possible 45-50Ahrs achieved by integrating the total 
current demand. The algorithms and linear approximations are therefore demonstrated to estimate the State-of-Health of a battery with good accuracy.

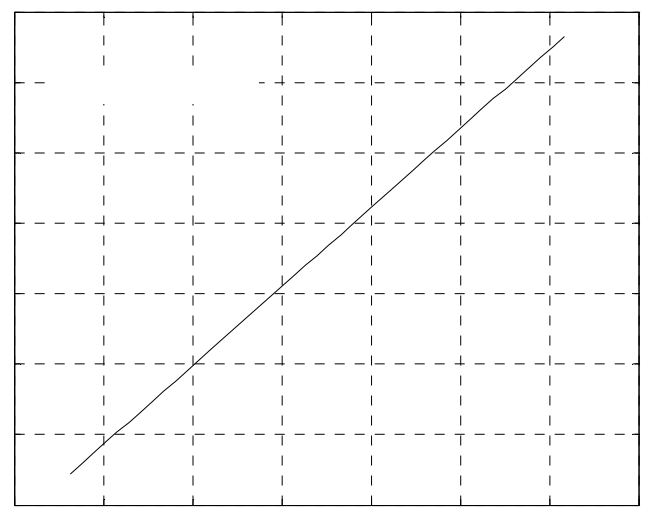

Figure 11. Relationship between estimated $\mathrm{C}_{\mathrm{b}}$ and available capacity

\section{CONCLUSION}

A novel modelling methodology of a battery for an allelectric Personal Rapid Transit vehicle is proposed consisting of Randles' circuit model, novel online Subspace parameter estimation and sliding-mode observer for the purpose of battery SoH/SoC Estimation. The use of Subspace estimation algorithms allows the sliding mode observer to adaptively estimate voltages associated with these functionality indicators even given erroneous initial conditions, so that ambient temperature effects and battery self-discharge issues are automatically compensated for. Moreover the resulting parameter estimations can identify fault conditions and areas of unsafe operation.

The voltages estimated by the observer are shown to facilitate the online monitoring of the model parameter associated with the main charge store of a battery, allowing degradation of the State-of-Health to be assessed, and more importantly, estimation of the available capacity of the battery under test over time.

\section{ACKNOWLEDGMENT}

The authors would like to thank the Engineering and Physical Sciences Research Council for supporting this work, whilst thanks also goes to Advanced Transport Systems Ltd for their cooperation, particularly Nicolas Zurlinden for providing the ULTra driving cycle data and press-shots.

\section{REFERENCES}

[1] http://www.atsitd.co.uk

[2] A. Jossen, "Fundamentals of battery dynamics", Journal of power souces, vol.154, p530-538, 2006

[3] D. Berndt, E. Meissner, R. Rusch, "Aging effects in valveregulated lead-acid batteries", 15th International Telecommunications Energy Conference (INTELEC), vol.2, p139-145, Sep. 1993

[4] P. Ruetschi, "Aging mechanisms and service life of lead-acid batteries", Journal of power souces, vol.127, p33-44, 2004

[5] B. S. Bhangu, P. Bentley, D. A. Stone, C. M. Bingham, "Nonlinear observers for predicting state-of-charge and state-ofhealth of lead-acid batteries for hybrid-electric vehicles," IEEE Transactions on Vehicular Technology, vol.54, no.3, p783-794, May 2005

[6] C. S. C. Bose, F. C. Laman, "Battery state of health estimation through coup de fouet," 22nd International Telecommunications Energy Conference (INTELEC), p597-601, 2000.

[7] Sinclair, R. Duke, S. Round, "An adaptive battery monitoring system for an electric vehicle," Proceedings. International Conference on Power Electronic Drives and Energy Systems for Industrial Growth, vol.2, p786-791, Dec. 1998

[8] Atsuo Kawamura, Takahiro Yanagihara, "State of charge estimation of sealed lead-acid batteries used for electric vehicles", 29th Annual IEEE Power Electronics Specialists Conference, vol.1, p583-587, May 1998

[9] Pyung-Soo Kim, "New estimation filtering for battery management systems of lead-acid cells in hybrid electric vehicles", International Journal of Computer Science and Network Security, vol.7, no.2, Feb. 2007"

[10] K. J. Vetter, "Elecktrochemische Kinetik", Springer, Berlin, p268, 1961

[11] L.Ljung, "System identification - Theory for the user", 2nd ed, PTR Prentice Hall, p317-353, 1999 